\title{
Bone Metabolism in Acromegaly
}

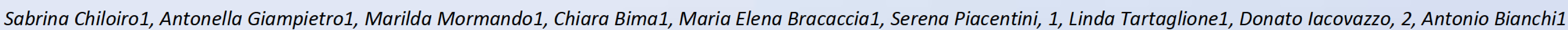
and Laura De Marinis 1

1 Università Cattolica del Sacro Cuore, Rome, Italy 2 Barts and The London School of Medicine, London, United Kingdom

\section{INTRODUCTION}

Over the recent past years, an expanding body of knowledge is supporting the idea that patients with acromegaly suffer from skeletal fragility and are at an increased risk for vertebral fractures. Fracture risk seems to correlate with the activity of the disease and the presence of concomitant additional risk factors, such as hypogonadism.

\section{AIM}

The aim of this paper was the evaluation of calcium and bone metabolism in a monocentric series of acromegaly patients, treated with pegvisomant alone or in association to long acting somatostatine analogs.

\section{MATERIALS AND METHODS}

\begin{abstract}
We retrospectively evaluated acromegaly patients in treatment with pegvisomant alone or in association with long acting somatostatine analogs (SSA). All the patient with at least 24 consecutive months pegvisomant treatment (alone or in combination with SSA) were included in the study. All the patients had been tested at least twice a year for biochemical acromegaly-disease evaluation and annually for calcium metabolism and for bone metabolism though serum test (calcium, phosphorus, 25-hydroxy vitamin D, parathyroid hormone, osteocalcin and b-crosslaps). In all the cases, BMD of femoral neck and lumbar spine was measured by DXA (Lunar Prodigy 8743, GEMedical System,MadisonWI, USA). Measurements were made at the time of the spinal X-ray. BMD is expressed as absolute values and Z-scores using a standard Caucasian European reference population. Fractured vertebrae, were excluded from the lumbar BMD analysis. A quantitative morphometrical assessment of VFs in the T4-L4 region had been performed using a dedicated morphometrical software (Spine-X Analyzer, ICAM Diagnostics, Milan, Italy). To compare qualitative variables, Fisher's exact test and the $\chi 2$ test were applied. To compare continuous variables, the T-student test was performed. Risk measures were described as odds ratios (ORs) with 95\% confidence intervals (Cls). Correlation between variables was sought using Pearson's correlation coefficient. Statistical significance was assumed when $\mathrm{p} \leq 0.05$. Data were analyzed using the SPSS Software, version 19.
\end{abstract}

\section{RESULTS}

\begin{abstract}
A total of 24 patients met the inclusion criteria. 8 were male (33.3\%). Mean age at acromegaly diagnosis was 39.3 years. 11 patients had a biochemically controlled acromegaly (45.8\%). Pegvisomant treatment was prescribed in 5 cases as monotherapy. A total of 16 fractures were documented and occurred in 8 patients. Mean spine BMD was 1.09 and mean femoral BMD was 0.9. Vertebral fracture, spine and femoral BMD and T-score are not influenced in our series from gender, GH receptor isoform expression, secondary hypogonadism, menopause, bone metabolism markers and hormonal replacement treatment for hypogonadism, hypoadrenalism and hypothyroidism. Instead, we found that biochemically acromegaly status strongly correlated with vertebrae facture events. In fact, a higher number of fracture were observed in not biochemically controlled acromegaly patients. Moreover, active acromegaly status correlated with a 10-folds higher risk of vertebrae fracture ( $p=0.012$, OR: 10.12 95\%IC 1.47-69.9). Moreover, in male acromegalic patient, fractures correlated also with lower serum testosterone ( $p=0.02$ ) and SHBG value $(p<0.001)$. In female acromegaly patient instead we failed to find any correlation between gonadic status and vertebrae fracture.
\end{abstract}
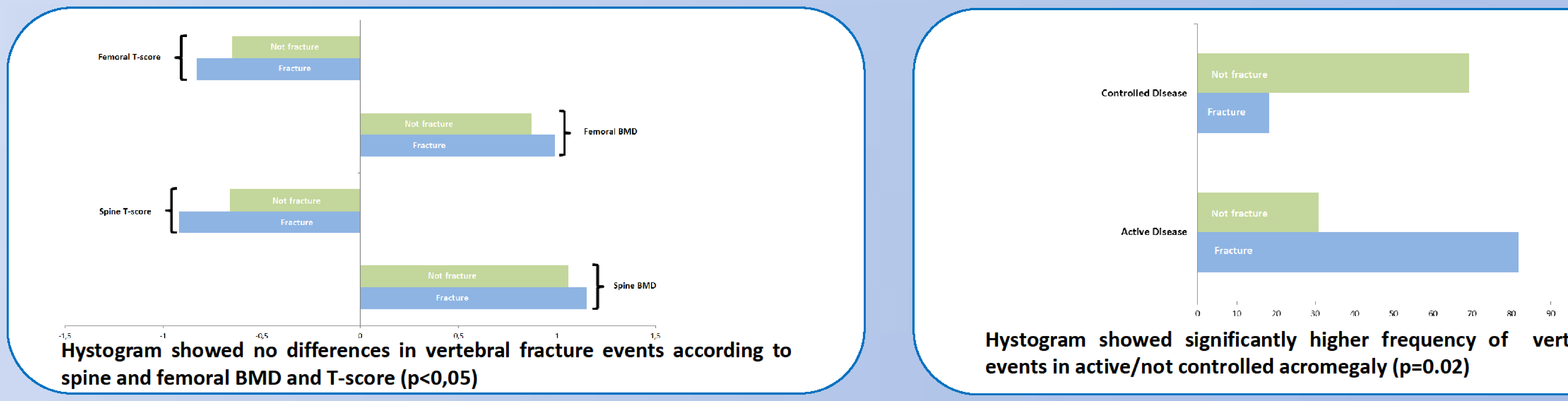

Hystogram showed significantly higher frequency of vertebral fracutere events in active/not controlled acromegaly $(p=0.02)$

\section{CONCLUSIONS}

Our study confirm the risk of vertebrae fractures in acromegaly and suggest that fracture risk is higher in not biochemically controlled acromegaly patients, particularly in males with low testosterone value. 